MODELING, IDENTIFICATION AND CONTROL, 1992, VOL. 13, NO. 3, 155-162

doi:10.4173/mic.19923.3

\title{
A method for closed loop automatic tuning of PID controllers $\dagger$
}

\author{
TOR STEINAR SCHEI \\ Keywords: Adaptive control, closed-loop systems, describing functions, identification, \\ industrial control, limit cycles, Nyquist criterion, PID control, process control.
}

\begin{abstract}
A simple method for the automatic tuning of PID controllers in closed loop is proposed. A limit cycle is generated through a nonlinear feedback path from the process output to the controller reference signal. The frequency of this oscillation is above the crossover frequency and below the critical frequency of the loop transfer function. The amplitude and frequency of the oscillation are estimated and the control parameters are adjusted iteratively such that the closed loop transfer function from the controller reference to the process output attains a specified amplitude at the oscillation frequency.
\end{abstract}

\section{Introduction}

In recent years several 'simple' methods for the automatic tuning of PID controllers have been proposed. A specific input to the process is usually generated automatically and the PID-parameters are then determined directly from the response. Many of these methods originate from the well known work of Ziegler and Nichols $(1942,1943)$. In their step response method the controller design is based on a registration of the openloop step response of the process, which is characterized by two parameters. An extension to the Ziegler-Nichols method is presented by Nishikawa et al. (1984). In this method the parameters of simple process models are determined directly from the step or pulse response. The model parameters are computed from certain areas related to the response and the PID parameters are determined from these model parameters. This transient response method is less sensitive to noise than the original step response method proposed by Ziegler and Nichols.

The Ziegler-Nichols frequency response method for tuning of PID controllers is based on the determination of a single point on the Nyquist curve for the process transfer function. This point is the intersection of the Nyquist curve with the negative real axis, which is traditionally called the critical point. The control parameters are then determined from the knowledge of the critical gain and the critical period. In the original Ziegler-Nichols method the critical point is determined by increasing the gain of a proportional controller until the control loop reaches the edge of stability. The critical point is then defined by the period and the amplitude of the oscillation generated in the control loop. This method is difficult to automate. Åström and Hägglund (1984a, 1984b) approximately determine the critical point by connecting a relay in the feedback loop from the process output to the process input. Most processes

Received 6 February 1992.

$\dagger$ Reprinted with permission from IFAC from the May 1992 issue of Automatica. An earlier version of this paper appeared in Actes de ECC ' 91 published by Hermes, Paris, July 1991.

$\ddagger$ SINTEF Automatic Control, 7034 Trondheim-NTH, Norway. 
will then oscillate in a limit cycle. The critical gain and the critical period are determined from the amplitude and the frequency of this oscillation.

These and similar methods have been used by the process control industry for the development of a wide range of 'autotuning' instruments that recently have appeared in the market (Morris 1987). References to other approaches are given by Gawthrop and Nomikos (1990).

The present method may be regarded as an extension of the Åström and Hägglund method to closed loop systems. A limit cycle is generated such that the control system oscillates at a frequency that is crucial for determination of control system stability and performance. The control parameters are adjusted iteratively such that the closed loop transfer function from the controller reference to the process output attains a specified amplitude at the oscillation frequency. It is assumed that a stable (and conservative) controller is established prior to the tuning, and the purpose of the proposed method is to improve the performance. For processes that are open-loop stable, conservative controllers might often be established by simple transient response experiments.

The paper is organized as follows. In Section 2 the excitation method is described under the assumptions that the PID parameters are constant and that a limit cycle is actually generated in the control system. Section 3 describes an iterative method for determination of the PID parameters. Simulation experiments are presented in Section 4. The advantages and disadvantages of the proposed method compared to the Åström and Hägglund open-loop autotuner are discussed in Section 5.

\section{The excitation method}

The system is excited by connecting a relay and a linear dynamic element in a feedback path from the process output to the reference signal for the controller as shown in Fig. 1. $M_{r}(\mathrm{~s})$ is the closed loop transfer function from the controller reference, $y_{r}$, to the process output, $y$. For most systems a limit cycle will be generated due to the nonlinear characteristic of the relay. The linear block $d(s)$ is used to influence the frequency of this limit cycle oscillation. The reference signal will vary in steps between $y_{0}-\Delta y$ and $y_{0}+\Delta y$, where $\Delta y$ is the amplitude of the relay function. The system will oscillate at approximately the frequency $\omega_{l c}$, where

$$
\angle d\left(j \omega_{l c}\right) M_{r}\left(j \omega_{l c}\right)=-180^{\circ}
$$

This follows from a describing function approximation of the relay characteristic.

In a PID controller the derivative action is normally applied to the measurement signal only, not to the reference signal. The controller transfer function from reference to controller output is then

$$
C_{r}(s)=K_{p}\left(1+\frac{1}{T_{i} s}\right)
$$

and the transfer function from measurement signal to controller output is

$$
-C_{y}(s)=-K_{p}\left(1+\frac{1}{T_{i} s}+\frac{T_{d} s}{1+\left(T_{d} / N_{f}\right) s}\right)
$$

where $K_{p}$ is the proportional gain, $T_{i}$ is the integral time constant, and $T_{d}$ is the derivative time constant. It is assumed that the derivative part is filtered by a first order lag with time constant $T_{d} / N_{f}$. The total controller output is

$$
u(s)=C_{r}(s) y_{r}(s)-C_{y}(s) y(s)
$$




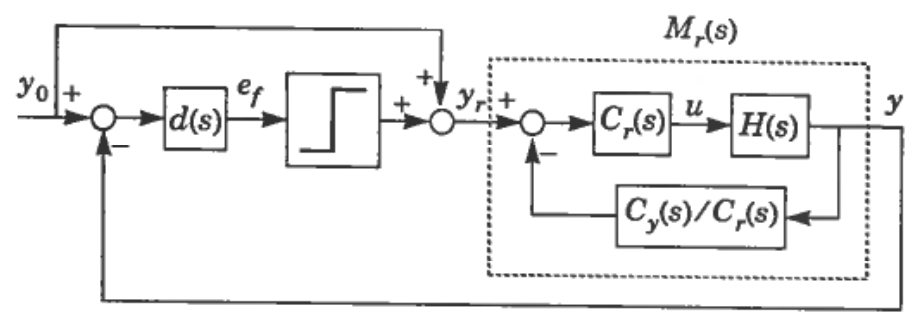

Figure 1. Block diagram showing the relay feedback path from the process measurement, $y$, to the reference signal for the controller, $y_{r}$.

The complementary sensitivity for the control loop is defined as

$$
M(s)=\frac{G(s)}{1+G(s)}, \quad G(s)=H(s) C_{y}(s)
$$

$G(s)$ is the loop transfer function and $H(s)$ is the transfer function for the process. The closed loop transfer function from controller reference to process output is

$$
M_{r}(s)=\frac{y(s)}{y_{r}(s)}=\frac{H(s) C_{r}(s)}{1+H(s) C_{y}(s)}=\frac{C_{r}(s)}{C_{y}(s)} M(s)
$$

The filter $d(s)$ is chosen to be

$$
d(s)=\frac{C_{y}(s)}{s C_{r}(s)}
$$

Equations (6) and (7) are inserted in (1):

$$
\angle d\left(j \omega_{l c}\right) M_{r}\left(j \omega_{l c}\right)=\angle \frac{M\left(j \omega_{l c}\right)}{j \omega_{l c}}=-180^{\circ}
$$

Hence, the frequency of the limit cycle, $\omega_{l c}$, is determined by

$$
\angle M\left(j \omega_{l c}\right)=-90^{\circ}
$$

The frequency of this oscillation is above the crossover frequency (gain equal one) and below the critical frequency (phase equal $-180^{\circ}$ ) for the loop transfer function. This can be seen from the Nichols chart in Fig. 2. The oscillation frequency is in the frequency range that is most crucial for the determination of stability and transient properties for the control system.

Some theoretical questions regarding existence and uniqueness of relay oscillations and accuracy of the describing function approximation are analysed by Åström and Hägglund (1984a) and Tsypkin (1984).

\section{Determination of PID parameters}

In the previous section the excitation method is described under the assumption that the control parameters are constant and that a stable limit cycle is generated in the control system. In the proposed tuning method the amplitude and frequency of this oscillation are determined by simple peak and zero-crossing detection of the signal $e_{f}(t)$ in Fig. 1.

The PID control parameters are updated iteratively from the following simple scheme. The amplitude of $M\left(j \omega_{l c}\right)$ is specified to be $m_{s-}$. The corresponding amplitude, 


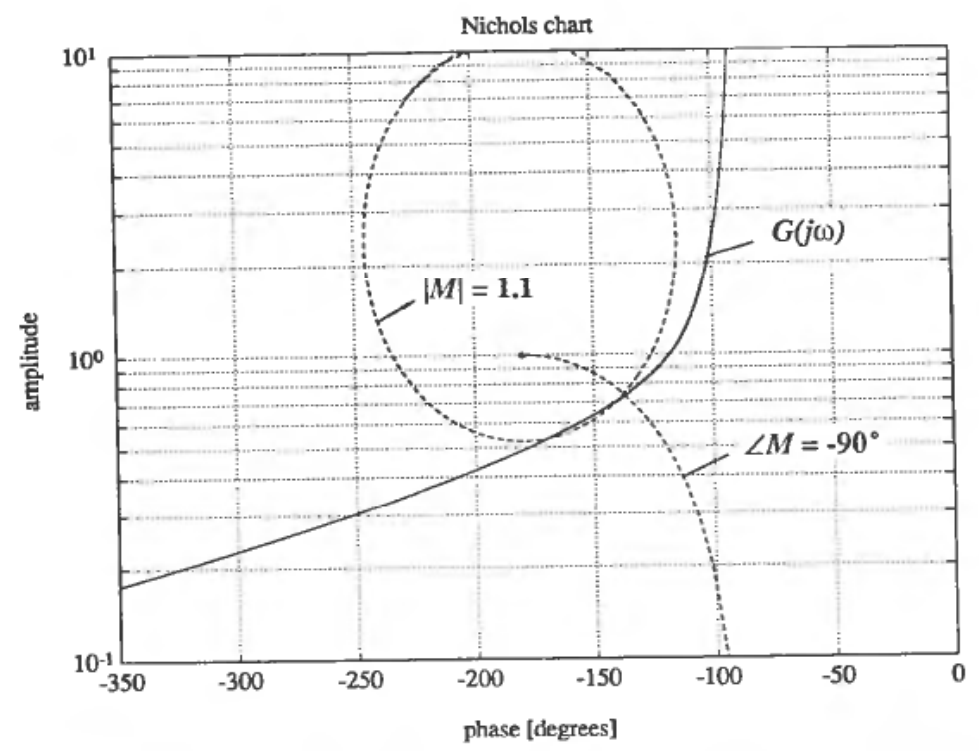

Figure 2. Nichols chart for determination of the complementary sensitivity function. Only the curves for $|M|=1 \cdot 1$ and $\angle M=-90^{\circ}$ are shown on the chart. The frequency and the amplitude of the limit cycle oscillation are determined by the intersection of the loop transfer function $G(j \omega)$ and the curve for $\angle M=-90^{\circ}$.

$g_{s}$, of the loop transfer function, $G\left(j \omega_{l c}\right)$, is determined from equation (5) and (9). At time $t_{i}$ the oscillation frequency is estimated to be $\hat{\omega}_{i}$, and the amplitude of $G\left(j \hat{\omega}_{i}\right)$ is estimated to be $\hat{g}_{i}=\hat{h}_{i}\left|C_{y, i}\left(j \hat{\omega}_{i}\right)\right|$, where $\hat{h}_{i}$ is the estimated process gain at the oscillation frequency. $C_{y, i}(s)$ is the transfer function for the controller from time $t_{i-1}$ to $t_{i}$. Now, the integral time constant, $T_{i}$, and the derivative time constant, $T_{d}$, are set proportional to $\hat{\omega}_{i}^{-1}$, and the proportional gain, $K_{p}$, is determined such that $\hat{h}_{i}\left|C_{y, i+1}\left(j \hat{\omega}_{i}\right)\right|=g_{s}$. $C_{y, i+1}(s)$ is the transfer function for the controller with the new parameter set. The tuning is terminated when the relative change in control parameters are below certain values.

The number of oscillations used for estimation of $\omega_{l c}$ and $\left|G\left(j \omega_{l c}\right)\right|$ is a compromise between stability of the autotuner and the duration of the tuning experiment. Simulations indicate that the control parameters might be updated once for each oscillation period. $t_{i-1}$ and $t_{i}$ then correspond to two zero-crossings of $e_{f}(t)$, with one oscillation period in between. The tuning algorithm might be made more robust against spurious disturbances by limiting the relative change in control parameters at each updating time.

In order to obtain the best possible tuning for a wide range of process characteristics, the autotuner has four modes. The 'tuning rules' below are determined through extensive simulation experiments with a wide range of process models.

Mode 1: $\quad T_{i}=\frac{3 \cdot 0}{\omega_{l c}}, \quad T_{d}=\frac{0 \cdot 75}{\omega_{l c}}, \quad m_{s}=1 \cdot 1$

Mode 2: $\quad T_{i}=\frac{3 \cdot 0}{\omega_{l c}}, \quad T_{d}=0, \quad m_{s}=1 \cdot 1$

Mode 3: $\quad T_{i}=\frac{1 \cdot 0}{\omega_{l c}}, \quad T_{d}=0, \quad m_{s}=0 \cdot 9$

Mode 4: $\quad T_{i}=\frac{6 \cdot 0}{\omega_{l c}}, \quad T_{d}=0, \quad m_{s}=1 \cdot 3$ 
Mode 1 and mode 2 are for PID and PI control with a medium value of the integral time constant. These modes work well for most processes. The third mode is for processes with very dominant non-minimum phase behaviour. The integral time constant should be chosen relatively short for these processes. The last mode is for control of integrating processes (e.g. level control). These processes should be controlled with a long integral time constant in order to obtain sufficient phase margin for the control loop.

\section{Simulation examples}

A PID controller is first tuned for the process model

$$
H(s)=\frac{1}{(1+30 s)(1+15 s)} \exp (-15 s)
$$

Figure 3 and 4 show the tuning experiment for a situation with no disturbances or measurement noise. The initial part of the tuning takes a relatively long time due to the very conservative initial control parameters. The open-loop transfer function $G(j \omega)$ after the tuning is shown in the Nichols diagram in Fig. 2. Now, the same model is simulated with white measurement noise and a periodic disturbance added to the process output. The standard deviation for the measurement noise is $1 \%$ and the disturbance is a sine wave with a period of 20 seconds and amplitude $1.5 \%$. A PI controller is tuned as shown in Fig. 5 and Fig. 6.

Other simulation experiments showing the performance of this method for various linear and nonlinear process models and for various disturbances are presented in Schei (1991).

\section{Conclusions}

A simple method for the automatic tuning of PID controllers has been proposed. The method is mainly investigated through extensive simulations with process models
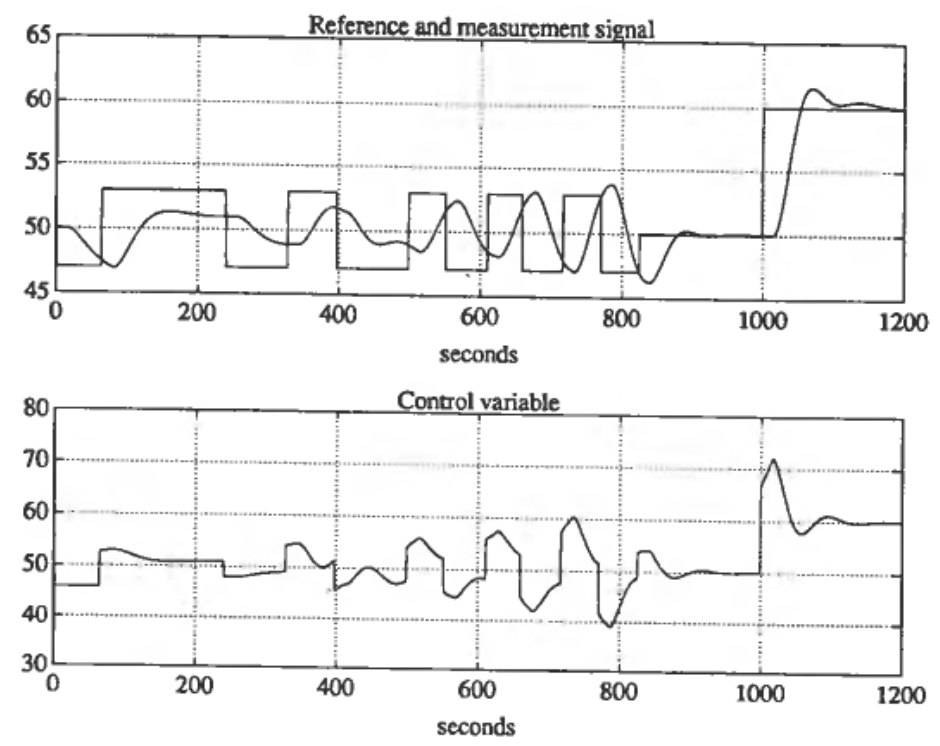

Figure 3. Autotuning of PID controller. The tuning is terminated after about 850 seconds. After 1000 seconds there is a step in the reference signal for the controller. 

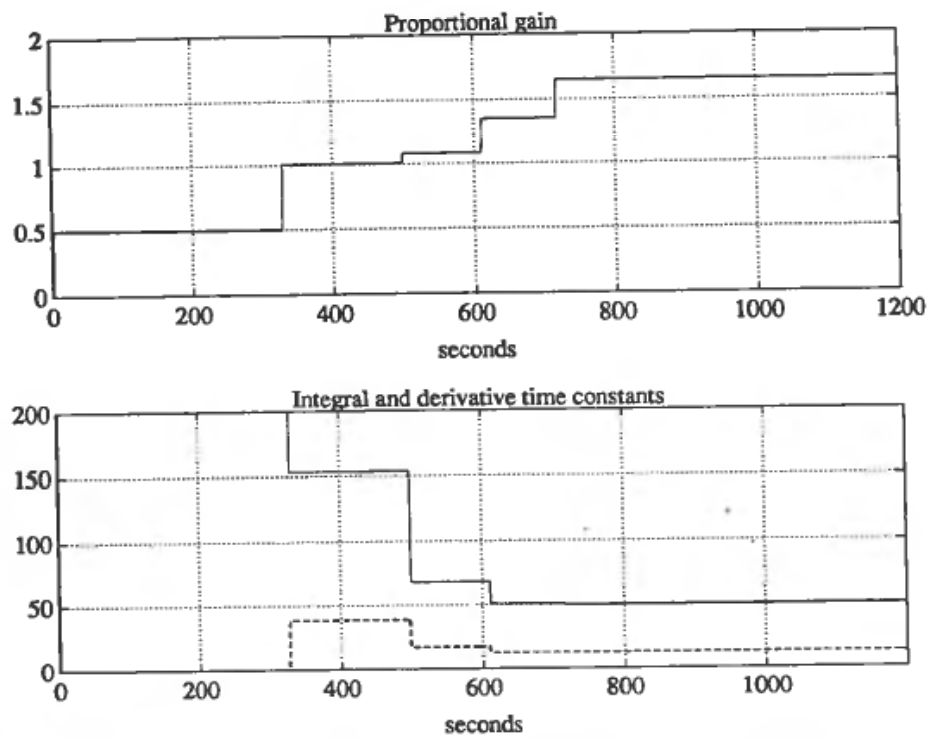

Figure 4. Proportional gain, integral time constant and derivative time constant (dashed line) during tuning of PID controller in Fig. 3. Initially the controller has only proportional action $\left(T_{i}=\infty\right.$ and $\left.T_{\mathrm{d}}=0\right)$.
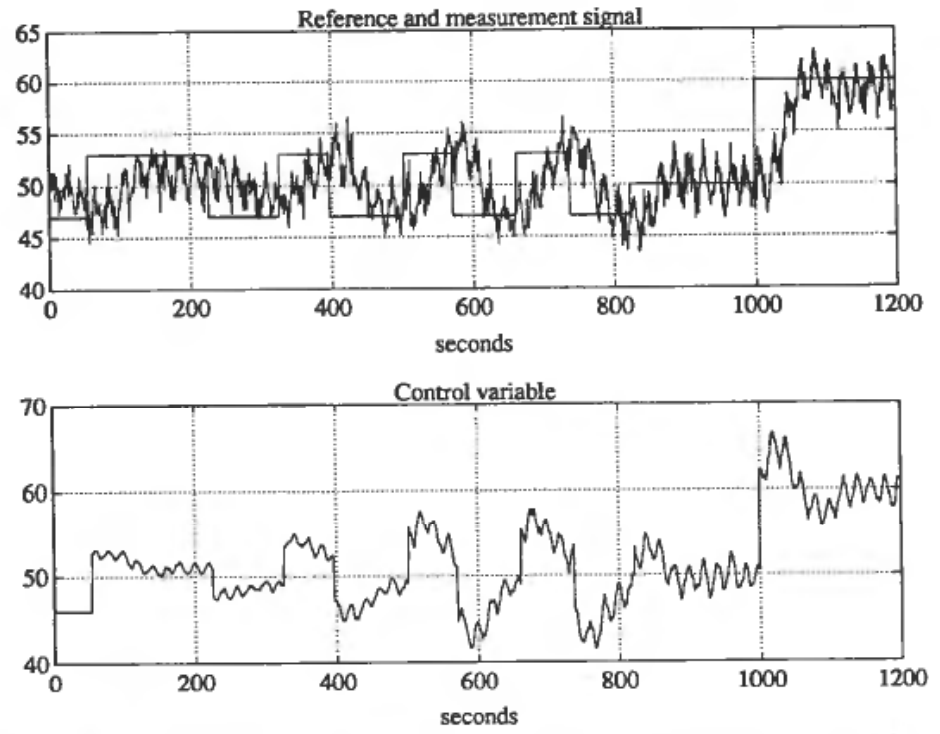

Figure 5. Autotuning of PI controller for process with measurement noise and periodic disturbance. 

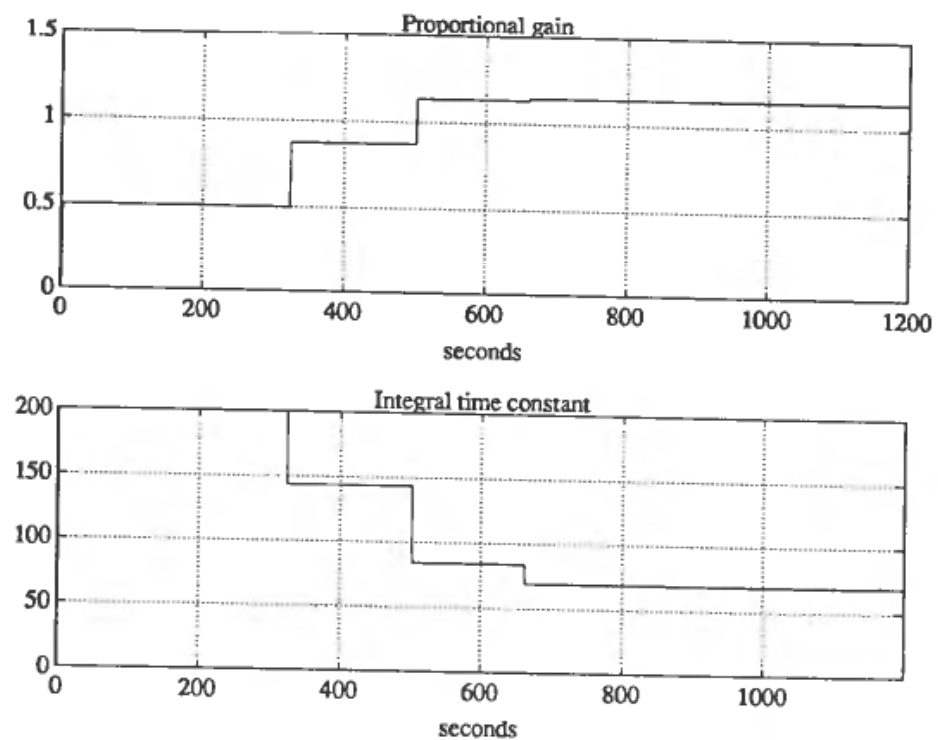

Figure 6. Proportional gain and integral time constant during tuning of PI controller in Fig. 5.

that are typical for the processes encountered in the process industry, and with different kinds of disturbances and measurement noise. The conclusion from these simulations is that the method performs very well if the tuning modes are reasonably chosen. Even for very difficult processes, where a PID controller is inferior to more advanced control methods, the controller tuning is often close to the 'best' that can be achieved within the limitations of a PID controller.

Compared to the Åström and Hägglund open-loop autotuner the proposed method is supposed to have the following properties. The oscillation frequency is reasonably chosen with respect to obtaining sufficient gain and phase margins for the control loop. With the Aström-Hägglund autotuner the process is excited at a frequency where the phase of the process transfer function is approximately $-180^{\circ}$. Often in process control it is not desirable to use derivative action in the controller. With a PI controller the phase lag of the loop transfer function then has to be more negative than $-180^{\circ}$. Hence, the oscillation frequency is above the critical frequency for the total control loop. In this situation it is assumed that the Åström-Hägglund autotuner has to be more conservative than the present method in order to ensure sufficient phase margin for the control loop.

The proposed method is also assumed to be less sensitive both to disturbances well above and well below the oscillation frequency compared to the Åström-Hägglund method. The former is due to the low pass character of the filter $d(s)$. The latter is due to the closed loop approach. In the Åström-Hägglund autotuner the process input vary in steps between two constant values. The step size then has to be chosen large enough to account for drift during the tuning.

The main disadvantage of the proposed method is assumed to be the duration of the tuning experiment. The proposed method is assumed to take longer time than the Åström-Hägglund method for two reasons. First, the excitation frequency is lower, especially if the initial controller tuning is very conservative. Second, the number of oscillation periods are probably higher due to the iterative updating of the control parameters. 


\section{ACKNOWLEDGMENTS}

I would like to thank Simrad Albatross for funding this work and particularly Sverre Gotaas and Helge Grøtterud for their strong interest in the practical application of advanced control methods. I would also like to thank my colleague Bjarne Foss for interesting discussions concerning this work.

\section{REFERENCES}

Åström, K. J. and HäGglund, T. (1984a). Automatic tuning of simple regulators. Proc. IF AC 9th World Congress, Budapest.

Åström, K. J. and HÄGGLUND, T. (1984b). Automatic tuning of simple regulators with specifications on phase and amplitude margins. Automatica, 20, 645-651.

GAwTHROP, P. J. and Nomikos, P. E. (1990). Automatic tuning of commercial PID controllers for single-loop and multiloop applications. IEEE Contr. Syst. Mag., 10, 34-42.

Morris, H. M. (1987). How adaptive are adaptive process controllers? Contr. Eng., March, 96-100.

NishiKaWA, Y., SANnOmiYa, N., OHTA, T. and TANaKA, H. (1984). A method for auto-tuning of PID control parameters. Automatica, 20, 321-332.

SCHEI, T. S. (1991). A new method for automatic tuning of PID control parameters. Proc. Europ. Contr. Conf., Grenoble, pp. 1522-1527.

TsYPKIN, Y. Z. (1984). Relay Control Systems. Cambridge University Press, U.K.

ZiEGLER, J. G. and NiCHOLS, N. B. (1942). Optimum settings for automatic controllers. Trans. ASME, 64, 759-768.

Ziegler, J. G. and Nichols, N. B. (1943). Process lags in automatic control circuits. Trans. ASME, 65, 433- 444. 